\title{
Barbijos quirúrgicos versus máscaras N95 para la prevención de infecciones respiratorias virales en trabajadores de la salud
}

\author{
Medical masks versus N95 respirators for preventing respiratory infection in healthcare workers
}

\section{Comentado de:}

Bartoszko JJ, et al. Influenza Other Respir Viruses. 2020;10.1111/irv. 12745. PMID: 32246890 ${ }^{1}$

\section{Introducción}

Los dispositivos de protección respiratoria son críticos para proteger a los trabajadores de la salud ante la enfermedad por el nuevo coronavirus SARS-CoV-2, denominada COVID-19. Sin embargo, las recomendaciones son contradictorias y los datos epidemiológicos sobre la efectividad de distintos tipos de máscaras contra COVID-19 es limitada.

\section{Objetivo}

Comparar los barbijos quirúrgicos con las máscaras N95 para prevenir infecciones virales confirmadas por laboratorio y enfermedades respiratorias, incluido el coronavirus específicamente, en trabajadores de la salud.

\section{Fuente de datos}

MEDLINE, Embase y CENTRAL, desde el 1 de enero de 2014 hasta el 9 de marzo de 2020 (actualización de la búsqueda publicada realizada del 1 de enero de 1990 al 9 de diciembre de $2014)^{2}$.

\section{Criterios de selección}

Se incluyeron ensayos controlados aleatorizados (ECA) que compararon el efecto protector de los barbijos quirúrgicos con las máscaras N95 en trabajadores de la salud.

\section{Recolección y análisis de datos}

Dos revisores seleccionaron los estudios, extrajeron los datos y evaluaron el riesgo de sesgo y la certeza de la evidencia de forma independiente.

\section{Resultados principales}

Cuatro ECA fueron incluidos en el meta-análisis, ajustando por conglomerados. El uso de barbijos quirúrgicos comparado con las máscaras N95, no aumentó la confirmación de laboratorio de infección respiratoria viral (incluyendo coronavirus) (Odds Ratio [OR] 1,06; Intervalo de Confianza [IC] del $95 \% 0,90$ a 1,25; heterogeneidad estimada mediante $\mathrm{I}^{2}=0 \%$; baja certeza en la evidencia) o la incidencia de enfermedad respiratoria clínica (OR 1,49 ; IC $95 \% 0,98$ a 2,28; $I^{2}=78 \%$; muy baja certeza en la evidencia). Solo un ECA evaluó a los coronavirus por separado y no se encontró diferencias entre los dos grupos $(p=0,49)$.

La confianza en los resultados para las infecciones por coronavirus es limitada debido a la imprecisión y a que la evidencia es indirecta.

\section{Conclusiones}

Evidencia de baja certeza sugiere que los barbijos quirúrgicos y los barbijos N95 ofrecen una protección similar contra la infección viral respiratoria, incluido el coronavirus, en trabajadores de la salud durante la atención que no genera aerosoles. En esta pandemia debe considerarse la preservación de los barbijos N95 para procedimientos de alto riesgo, que generan aerosoles, cuando éstos escaseen.

Fuente de financiamiento/Conflicto de interés de los autores: Este estudio se llevó adelante sin financiamiento externo. Los autores no repor taron conflictos de interés.

\section{Comentario}

En la actualidad se estima que 90.000 trabajadores de la salud han sido infectados en todo el mundo desde el inicio de la pandemia de COVID19 ${ }^{3}$. La necesidad de una adecuada protección de los profesionales que se exponen diariamente al SARS-CoV-2 se enfrenta con la limitada disponibilidad de los equipos de protección personal (EPP) en todos los ámbitos de atención, poniendo en alerta al sistema de salud. En este contexto, en el que resulta fundamental priorizar el cuidado de quienes se exponen a diario a la vez que promover una adecuada utilización de los recursos dada su posible escasez, resulta imprescindible tener respuestas a los interrogantes como el que se plantea en este artículo.

Existen recomendaciones diversas entre las distintas organizaciones dedicadas al cuidado de la salud. Por ejemplo, el Centro para el Control y la Prevención de Enfermedades de los EE.UU. (CDC, por sus iniciales en inglés) y el Centro Europeo para la Enfermedad y la Prevención (ECDC, por sus iniciales en inglés) recomiendan preferentemente la utilización de máscaras N95 para la atención de rutina de pacientes con COVID-19 sin aerolización de partículas ${ }^{4-6}$, mientras que, en simultáneo, la Organización Mundial de la Salud (OMS) y la Agencia de Salud Pública de Canadá recomiendan el uso de barbijos quirúrgicos ${ }^{7,8}$. En Argentina, el Ministerio de Salud de la nación recomienda al personal de salud que, para la asistencia de casos sospechosos y casos confirmados de COVID-19, utilice el barbijo quirúrgico y sólo ante la realización de procedimientos o maniobras que generen aerosoles, la máscara $\mathrm{N} 95^{9}$.

De acuerdo a la revisión sistemática resumida en este artículo, los barbijos quirúrgicos ofrecerían igual protección que las máscaras N95 para prevenir en los profesionales de la salud las infecciones respiratorias virales confirmadas por laboratorio durante la atención usual de pacientes y la realización de procedimientos no generadores de aerosoles, lo que avalaría las recomendaciones menos agresivas como la de la OMS para el uso racional de los recursos. Sin embargo, debido a la imprecisión de los resultados obtenidos en el meta-análisis, es decir, al amplio intervalo de confianza observado en las estimaciones, no se puede establecer de manera concluyente que no existan diferencias en el grado de protección que ofrecen los distintos dispositivos evaluados, y la inclusión de nuevos investigaciones en el futuro podría modificar sustancialmente 
estos hallazgos. Por otro lado, es necesario remarcar que este meta-análisis incluyó solo un ECA que estudió individualmente la transmisión de infección por coronavirus (no SARS-Cov-2), motivo por el cual no se pudo jerarquizar la protección que estos dispositivos proveen frente a la infección por el germen específico causante de la actual pandemia. Esto significa que los hallazgos mencionados pueden no ser generalizables al SARS-CoV-2. Estas consideraciones permitieron calificar como de baja o muy baja confianza a la evidencia obtenida.

\section{Conclusiones de la comentadora}

Probablemente en los próximos meses contemos con información más específica acerca del uso de EPP y su eficacia para proteger a los profesionales de la salud frente al SARS-CoV-2. Mientras tanto, es fundamental cumplir con las recomendaciones de las autoridades sanitarias nacionales y que estas aseguren la provisión y el acceso a los EPP de todo el personal en todas las jurisdicciones.

\section{Carolina Carrara [ Servicio de Medicina Familiar y Comunintaria, Hospital Italiano de Buenos Aires. carolina.carrara@hospitalitaliano.org.ar ]}

Carrara C Barbijos quirúrgicos versus máscaras N95 para la prevención de infecciones respiratorias virales en trabajadores de la salud. Evid Actual Pract Ambul. 2020;23(2):e002067. Comentado de: Bartoszko JJ, et al. Medical masks vs N95 respirators for preventing COVID-19 in healthcare workers: A systematic review and meta-analysis of randomized trials. Influenza Other Respir Viruses. 2020;00:1-9. PMID: 32246890

\section{Referencias}

1. Bartoszko JJ, Farooqi M, Alhazzani W, et al. Medical masks vs N95 respirators for preventing COVID-19 in healthcare workers: A systematic review and meta-analysis of randomized trials. Influenza Other Respir Viruses. 2020;Available from: 10.1111/irv.12745.

2. Smith JD, MacDougall CC, Johnstone J, et al. Effectiveness of N95 Respirators Versus Surgical Masks in Protecting Health Care Workers From Acute Respiratory Infection: A Systematic Review and Meta-Analysis. CMAJ. 2016;188(8):567-574. Available from: 10.1503/cmaj.150835.

3. International Council of Nurses. ICN calls for data on healthcare worker infection rates and deaths; 2020. Available from: https://www.icn.ch/news/ icn-calls-data-healthcare-worker-infection-rates-and-deaths [Last access: 2020-05-20].

4. Centers for Disease Control and Prevention (CDC). Interim Infection Prevention and Control Recommendations for Patients with Suspected or Confirmed Coronavirus Disease 2019 (COVID19) in Healthcare Settings March 10. Centers for Disease Control and Prevention (CDC). 2020;Available from: https://www.cdc.gov/coronavirus/2019-ncov/infection-control/control-recommendations.html.

5. European Centre for Disease Prevention and Control. Guidance for wearing and removing personal protective equipment in healthcare settings for the care of patients with suspected or confirmed COVID-19;2020. Available from: https://www.ecdc.europa.eu/sites/default/files/documents/COVID19-guidance-wearing-and-removing-personal-protective-equipment-healthcare-settings-updated.pdf.

6. European Centre for Disease Prevention and Control. Infection prevention and control for COVID-19 in healthcare settings - Third update. Stockholm; 2020. Available from: https://www.ecdc.europa.eu/en/publications-data/infection-prevention-and-control-and-preparedness-covid-19-healthcaresettings\#no-link.

7. World Health Organization. Rational use of personal protective equipment for coronavirus disease 2019 (COVID-19); 2020. Available from: https: //apps.who.int/iris/bitstream/handle/10665/331215/WHO-2019-nCov-IPCPPE use-2020.1-eng.pdf.

8. Public Health Agency of Canada (PHAC). Coronavirus disease (COVID-19): For health professionals; 2020. Available from: https://www.canada.ca/ en/public-health/services/diseases/2019-novel-coronavirus-infection/health-professionals.html\#i.

9. Recomendaciones para el uso de los EPP; 2020. Available from: https://www.argentina.gob.ar/salud/coronavirus-COVID-19/recomendaciones-usoepp [Last access: 2020-05-21]. 